\title{
CONSENSUS FOR THE DISTRIBUTED ESTIMATION OF POINT DIFFUSION SOURCES IN SENSOR NETWORKS
}

\author{
John Murray-Bruce and Pier Luigi Dragotti \\ Imperial College London \\ Electrical and Electronic Engineering Department \\ e-mail:\{john.murray-bruce07, p.dragotti\}eimperial.ac.uk
}

\begin{abstract}
In this contribution, we implement a fully distributed diffusion field estimation algorithm based on the use of average consensus schemes. We show that the field reconstruction problem is equivalent to estimating the sources of the field, and then derive an exact inversion formula for jointly recovering these sources when they are localized and instantaneous. Next we adapt this formula to the sensor network setting when only spatiotemporal samples of the field are available, and only local interactions between the sensors are allowed. To this end, we propose a robust distributed algorithm for reconstructing two-dimensional diffusion fields, sampled with a network of arbitrarily placed sensors. The proposed distributed algorithm is validated through numerical simulations in the noisy, multiple source setting.
\end{abstract}

Index Terms - Sensor networks, diffusion fields, spatiotemporal sampling and reconstruction, distributed averaging, consensus

\section{INTRODUCTION}

The use of sensor networks for monitoring physical fields is becoming increasingly important. We consider sensor networks for the monitoring of diffusion fields. Such fields are encountered in several physical and biological situations, from the variation of temperature in fluids and disease epidemic dynamics to the release of nuclear and bio-chemical substances. However, diffusion fields are nonbandlimited and so require a dense set of spatiotemporal samples in order to recover the field. Consequently, considerable research efforts have been made towards developing robust sensor data fusion schemes. Several centralized approaches for diffusion fields like [1-7] exist. However these schemes are vulnerable to single point failure, specifically the network becomes unoperational if the fusion center fails. Moreover, communicating with the fusion center typically require long range transmissions from the sensor nodes, which can lead to huge communication costs and bottlenecks. As a result efforts by the signal processing community have aimed to decentralize, and fully distribute, the field estimation problem. For instance, $\mathrm{Lu}$ and Vetterli propose a distributed adaptive sampling scheme [8], van Waterschoot and Leus [9] develop a distributed scheme based on finite element method. In addition, a distributed field reconstruction method using hybrid shift-invariant spaces is proposed in [10], whilst a distributed extension of standard compressed sensing techniques is developed in [11].

The main task of this paper therefore is to derive a noise robust, fully distributed sensor data fusion scheme for reconstructing

This work is supported by the European Research Council (ERC) starting investigator award Nr. 277800 (RecoSamp). diffusion fields driven by localized sources. To achieve this, we first argue that the field estimation problem is equivalent to estimating the sources of the field. Then we extend the sensing functions approach of [12] used for simultaneously recovering all unknown source parameters (intensities, locations and activation times) of the field, to the case of distributed estimation. In contrast to [12] which requires a centralized estimation, we show that this computation can be distributed using a modification of the distributed gossip algorithms for average consensus, such that each sensor in the network only needs to exchange some properly modified versions of its sensor measurements to its neighboring nodes. This allows each sensor to converge to the true values of a specific family of integrals and hence successfully recover the unknown sources. Although the focus of this paper is on the recovery of instantaneous sources, the theory developed herein can also be extended to the time-varying source distribution considered in [13].

The paper is organized as follows. In Section 2 the distributed sampling and reconstruction problem in the source estimation setting is formalized, along with assumptions on the model of the sensor network. Section 3 presents an overview of gossip algorithms for distributed consensus. We then present a derivation of the consensus based distributed source estimation scheme in Section 4 for simultaneous recovery of multiple instantaneous and localized sources. Numerical simulations are given in Section 5 and concluding remarks in Section 6.

\section{PROBLEM FORMULATION}

In this paper, we are specifically concerned with the distributed reconstruction of two-dimensional diffusion fields from its irregular spatial samples obtained using a network of sensors (see Figure 1). It is well-known that the diffusion field $u(\mathbf{x}, t)$, at a location $\mathbf{x} \in \mathbb{R}^{2}$ and time $t$, induced by the unknown source distribution $f(\mathbf{x}, t)$ will propagate through space and time according to the diffusion equation,

$$
\frac{\partial}{\partial t} u(\mathbf{x}, t)=\mu \nabla^{2} u(\mathbf{x}, t)+f(\mathbf{x}, t)
$$

where $\mu$ is the diffusivity of the medium through which the field propagates. Furthermore, the Green's function solution of this PDE is such that:

$$
u(\mathbf{x}, t)=(g * f)(\mathbf{x}, t),
$$

where $g(\mathbf{x}, t)=\frac{1}{4 \pi \mu t} e^{-\frac{\|\mathbf{x}\|^{2}}{4 \mu t}} H(t)$ is the Green's function of the diffusion field, and $H(t)$ is the unit step function. Clearly if $f(\mathbf{x}, t)$ is known precisely, (2) allows us to perfectly reconstruct the field $u(\mathbf{x}, t) \forall \mathbf{x}, t$. Hence, we will concentrate on estimating the source distribution $f$ using sensor networks. We consider diffusion fields induced by spatially localized and temporally instantaneous sources, 
with the following distribution:

$$
f(\mathbf{x}, t)=\sum_{m=1}^{M} c_{m} \delta\left(\mathbf{x}-\boldsymbol{\xi}_{m}, t-\tau_{m}\right),
$$

where $c_{m}, \tau_{m} \in \mathbb{R}$ and $\boldsymbol{\xi}_{m} \in \Omega$ are the intensity and activation time and spatial location of the $m$-th source respectively, and $\boldsymbol{\xi}_{m}=$ $\left(\xi_{1, m}, \xi_{2, m}\right)$. We seek a distributed estimation strategy for solving this estimation problem. So that each sensor performs local data acquisition (samples the field) and then through localized interactions (i.e. exchanging properly modified versions of its measurements with its neighbors) estimates the unknown parameters $\left\{c_{m}, \boldsymbol{\xi}_{m}, \tau_{m}\right.$ : $m=1, \ldots, M\}$ of the field and hence reconstruct the field. The distributed sampling and reconstruction problem is summarized below for clarity:

$\mathcal{P}$ : Let $\mathcal{S}=\{1, \ldots, N\}$ denote a network of $N$ sensors with each sensor $n$ located arbitrarily at $\mathbf{x}_{n}$ having access to local samples $\varphi_{n}\left(t_{l}\right)=u\left(\mathbf{x}_{n}, t_{l}\right)$ of the field $u$, at times $t_{l}$ for $l=0,1, \ldots, L$. Given only these local temporal samples, we intend to estimate $\left\{c_{m}, \boldsymbol{\xi}_{m}, \tau_{m}: m=1, \ldots, M\right\}$ by performing local exchange of messages with neighboring nodes.

\subsection{System Model and Assumptions}

The sensor network is assumed to be a strongly connected random geometric graph (RGG) $\mathcal{G}\left(N, r_{c o n}\right)$, with $N$ nodes and connectivity radius $r_{c o n}$. This is obtained by placing $N$ nodes uniformly at random over a square region and then placing an edge between two nodes if their Euclidean distance is at most $r_{c o n}$. The communication between nodes is assumed to be noiseless and much faster than the sampling rate. Sensors are synchronized, so that the field samples obtained by the $n$-th sensor is $\left\{\varphi_{n}\left(t_{l}\right)\right\}_{l=0}^{L}$. Furthermore, upon deployment of the sensors an initialization process is initiated, where: $a$ ) the sensors learn the topology of the network; and $b$ ) they each perform the Delaunay triangulation(see Figure 1) such that we obtain a graph $\mathcal{G}_{\text {del }}=(\mathcal{V}, \mathcal{E})$, with the vertex set $\mathcal{V}$ corresponding to the locations of the sensors and $\mathcal{E}$ are the edges of the triangulation. Hence every sensor $n$ knows if it lies on the convex hull boundary of the triangulation or in the interior of the convex hull; and also the total number $J_{n}$ of triangles for which it is a vertex, as well as their areas $\left|\Delta_{n, j}\right|$ for $j=1, \ldots, J_{n}$.

\section{GOSSIP ALGORITHMS OVERVIEW}

Gossiping $[14,15]$ is a distributed strategy for achieving consensus amongst agents in a network through a local exchange of data. Following the early works of [14] in the area, it has gained considerable interest for in-network processing in sensor networks as it mitigates the need for specialized routing protocols. In addition, gossip-based algorithms are robust to bottlenecks and link failures making it suitable for our distributed estimation problem. In our simulation results we use the archetypal pairwise randomized gossip algorithm [16], but stress that the results derived in this paper can be immediately extended to other gossiping schemes, such as $[17,18]$. An in depth survey of gossiping algorithms in sensor networks is given in [15].

In randomized pairwise gossip, each node preserves an estimate of the sum and hence average of the node values. Let the value of node $n$ after the $i$-th pairwise gossip round be $y_{n, i}$, hence $y_{n, 0}$ is its initial value. In an iteration, a node $n$ selected uniformly at random wakes up and contacts a randomly selected neighbor $n^{\prime}$ within its connectivity radius, and they both update their estimates by setting $y_{n, i+1}=y_{n^{\prime}, i+1}=\left(y_{n, i}+y_{n^{\prime}, i}\right) / 2$. Under this scheme it can be shown that, if a network (of $N$ nodes) is connected and each

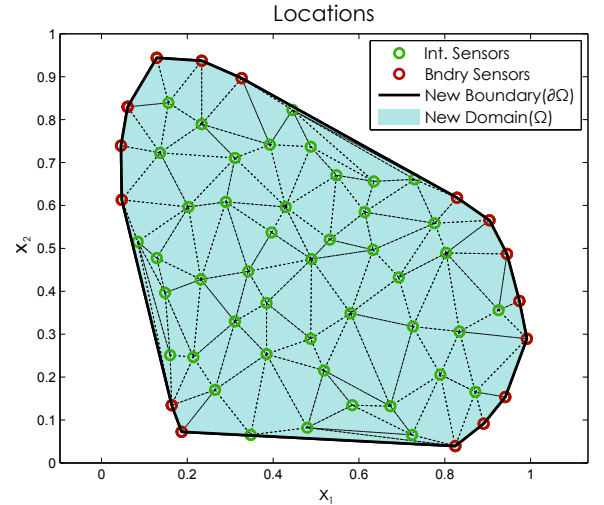

Fig. 1: An arbitrary sensor placement - the monitored domain $\Omega$ divided into triangular meshes and the domain boundary $\partial \Omega$ divided into straight line segments (black solid lines).

pair of nodes communicate often enough the estimate of each node converges to the global network average $\bar{y}=\frac{1}{N} \sum_{n=1}^{N} y_{n, 0}$. Performance guarantees and convergence results have also been studied (see [16] and the references therein).

The localized interactions in our field estimation setting will be based on the use of gossip algorithms for the distributed computation of a family of integrals whose final values can be used to reveal the unknown source parameters. In the following section we present our strategy for estimating these family of integrals through localized interactions alone and then we demonstrate how to recover the unknown source parameters.

\section{DIFFUSION SOURCE ESTIMATION}

In this section, we extend the sensing function approach of $[12,19]$ introduced for joint recovery of intensities and locations of multiple localized and instantaneous diffusion sources, to the case of distributed recovery. We overview the centralized recovery, before demonstrating how the computations can be distributed amongst the sensor nodes using consensus-based schemes.

\subsection{Joint Intensity, Location and Activation Time Recovery}

Green's second theorem allows us to relate the evolution of the field $u$ along a boundary $\partial \Omega$ to the field inside it as follows:

$$
\oint_{\partial \Omega}\left(\Psi_{k} \nabla u-u \nabla \Psi_{k}\right) \cdot \hat{\mathbf{n}}_{\partial \Omega} \mathrm{d} S=\int_{\Omega}\left(\Psi_{k} \nabla^{2} u-u \nabla^{2} \Psi_{k}\right) \mathrm{d} V,
$$

where $\hat{\mathbf{n}}_{\partial \Omega}$ is the outward pointing unit normal vector to the boundary $\partial \Omega, \Omega$ is the bounded region and $\Psi_{k}$ is a sensing function. If $\Psi_{k}$ is chosen to satisfy $\frac{\partial \Psi_{k}}{\partial t}+\mu \nabla^{2} \Psi_{k}=0$, then (4) reduces to,

$$
\int_{\Omega} \frac{\partial}{\partial t}\left(u \Psi_{k}\right) \mathrm{d} V-\mu \oint_{\partial \Omega}\left(\Psi_{k} \nabla u-u \nabla \Psi_{k}\right) \cdot \hat{\mathbf{n}}_{\partial \Omega} \mathrm{d} S=\int_{\Omega} \Psi_{k} f \mathrm{~d} V,
$$

by substituting (1) and $\nabla^{2} \Psi_{k}=-\frac{1}{\mu} \frac{\partial \Psi_{k}}{\partial t}$ into (4) and rearranging. Now before integrating (5) over $t \in[0, T]$ as in [12], we first multiply through by a time-varying sensing function $\Gamma(t)$ and then time-integrate to obtain,

$$
\mathcal{R}(k)=\left\langle\Psi_{k}(\mathbf{x}) \Gamma(t), f\right\rangle=\int_{\Omega} \int_{t} \Psi_{k}(\mathbf{x}) \Gamma(t) f(\mathbf{x}, t) \mathrm{d} t \mathrm{~d} V
$$


where for a time-independent choice of $\Psi_{k}$,

$$
\mathcal{R}(k) \stackrel{\text { def }}{=} \int_{\Omega}\left(\Psi_{k} \dot{U}\right)(\mathbf{x}, T) \mathrm{d} V-\mu \oint_{\partial \Omega}\left(\Psi_{k} \nabla U-U \nabla \Psi_{k}\right) \cdot \hat{\mathbf{n}}_{\partial \Omega} \mathrm{d} S,
$$

with $U(\mathbf{x}, T)=\int_{0}^{T} \Gamma(t) u(\mathbf{x}, t) \mathrm{d} t$ and $\dot{U}(\mathbf{x}, T)=\Gamma(T) u(\mathbf{x}, T)-$ $\int_{0}^{T} \frac{\partial \Gamma}{\partial t} u(\mathbf{x}, t) \mathrm{d} t$. Subsequently, define $\Phi_{n}\left(t_{L}\right) \stackrel{\text { def }}{=} U\left(\mathbf{x}_{n}, t_{L}\right)$, similarly $\dot{\Phi}_{n}\left(t_{L}\right) \stackrel{\text { def }}{=} \dot{U}\left(\mathbf{x}_{n}, t_{L}\right)$.

Furthermore, for the source parameterization (3), the right hand side of (6) becomes, $\mathcal{R}(k)=\sum_{m=1}^{M} c_{m} \Gamma\left(\tau_{m}\right) \Psi_{k}\left(\boldsymbol{\xi}_{m}\right)$. If we choose $\Psi_{k}(\mathbf{x})$ to be analytic and $\Gamma(t)$ to be a time-varying function, specifically $\Psi_{k}(\mathbf{x})=e^{-k\left(x_{1}+\mathrm{j} x_{2}\right)}$ and $\Gamma(t)=e^{-\mathrm{j} t / T}$ for stability reasons, then the Vandermonde system

$$
\mathcal{R}(k)=\sum_{m=1}^{M} c_{m} e^{-\mathrm{j} \tau_{m} / T} e^{-k\left(\xi_{1, m}+\mathrm{j} \xi_{2, m}\right)} k=0,1, \ldots, K,
$$

where $\mathrm{j}=\sqrt{-1}$, is obtained. Finally (8) can be used to solve for all $M$ tuples $\left(c_{m} e^{-\mathbf{j} \tau_{m} / T}, \boldsymbol{\xi}_{m}\right)$ using Prony's method [20,21], provided $K \geq 2 M-1$. Having seen how to recover all unknown source parameters simultaneously given the integral measurements (7), we are now left with obtaining this family of integral measurements in a distributed way.

\subsection{Source Estimation: A Consensus Approach}

Given access to all spatiotemporal sensor measurements of the field $\left\{\varphi_{n}\left(t_{l}\right): l=0,1, \ldots, L\right\}_{n=1}^{N}$ at a fusion center for example, it is possible to compute reliable estimates of (7) using standard quadrature techniques as discussed in [12], and hence recover the unknown source parameters using Prony's method. In this contribution, we intend to distribute the estimation of $\{\mathcal{R}(k)\}$ using (7) across all sensors.

Proposition 1. By exchanging the properly weighted sum of sensor measurements, $y_{n}(k)=N\left[A_{n}(k) \dot{\Phi}_{n}\left(t_{L}\right)-\mu B_{n}(k) \Phi_{n}\left(t_{L}\right)\right]$ where $A_{n}(k)$ and $B_{n}(k)$ are dependent on $\Psi_{k}$ and the topology of the network, with neighboring sensors it is possible for each sensor $n$ to recover the unknown source parameters.

Proof. Firstly, consider the surface integral contribution in (7), if the bounded domain $\Omega$ is partitioned into non-overlapping triangular subdivisions $\left\{\Delta_{j}\right\}_{j=1}^{J}$ such that $\bigcup_{i=1}^{I} \Delta_{i}=\Omega$ and $\Delta_{i} \bigcap \Delta_{j}=\emptyset$ for $i \neq j$, then this integral can be approximated by the sum[22]:

$$
\int_{\Omega}\left(\Psi_{k} \dot{U}\right)(\mathbf{x}, T) \mathrm{d} V \approx \frac{1}{3} \sum_{j=1}^{J} \sum_{j^{\prime}=1}^{3} \Psi_{k}\left(\mathbf{v}_{j, j^{\prime}}\right) \dot{\Phi}_{j, j^{\prime}}\left(t_{L}\right)\left|\Delta_{j}\right|,
$$

where $\mathbf{v}_{j, j^{\prime}}$ is the $j^{\prime}$-th vertex of triangle $j$-th and $\dot{\Phi}_{j, j^{\prime}}\left(t_{L}\right)=$ $\dot{U}\left(\mathbf{v}_{j, j^{\prime}}, t_{L}\right)$ is the measurement of the sensor situated at this vertex at time $t=t_{L}=T$. Moreover this double sum, in (9), can be reduced to the single sum:

$$
\int_{\Omega}\left(\Psi_{k} \dot{U}\right)(\mathbf{x}, T) \mathrm{d} V \approx \sum_{n=1}^{N} \underbrace{\left(\frac{1}{3} \Psi_{k}\left(\mathbf{x}_{n}\right) \sum_{j=1}^{J_{n}}\left|\Delta_{n, j}\right|\right)}_{:=A_{n}(k)} \dot{\Phi}_{n}\left(t_{L}\right),
$$

where $\Delta_{n, j}$ and $\left|\Delta_{n, j}\right|$ are used to denote the $j$-th triangle, and its area respectively, of which node $n$ is a vertex. This equality follows by noticing that $\dot{\Phi}_{j, j^{\prime}}\left(t_{L}\right)$ is always multiplied by the area of the $j$-th triangle and $1 / 3 \Psi_{k}\left(\mathbf{v}_{j, j^{\prime}}\right)$. Hence denoting the set of all triangles that share a common vertex $n$ located at $\mathbf{x}_{n}$ by $\mathcal{T}_{n}=\left\{\Delta_{n, 1}, \Delta_{n, 2}, \ldots, \Delta_{n, J_{n}}\right\}$, the measurement $\dot{\Phi}_{n}\left(t_{L}\right)$ is always weighted by the sum of the areas of its corresponding triangles and $1 / 3 \Psi_{k}\left(\mathbf{x}_{n}\right)$. We denote this weight that directly depends on the sensing function and Delaunay triangulation (equivalently, the topology of the network) by $A_{n}(k)$.

For the boundary integral, the time-integrated field $U(\mathbf{x})$ and its spatial derivative $\nabla U(\mathbf{x})=\left[\frac{\partial U}{\partial x_{1}}, \frac{\partial U}{\partial x_{2}}\right]^{\top}=\left[U_{x_{1}}, U_{x_{2}}\right]^{\top}$ are required. Let $\mathcal{S}_{\partial \Omega}=\{1, \ldots, n, \ldots, I\}$ denote the cyclically ordered set of the boundary sensors, these coincide with the vertices of the Convex Hull. Furthermore, assume the elements of $\mathcal{S}_{\partial \Omega}$ are in counterclockwise order, then: $\hat{\mathbf{n}}_{\partial \Omega} \mathrm{d} S \approx\left[x_{2, n}-x_{2, n-1}, x_{1, n-1}-x_{1, n}\right]^{\top}$. Hence the boundary integral can be approximated as follows:

$$
\begin{aligned}
& \oint_{\partial \Omega}\left(\Psi_{k} \nabla U-U \nabla \Psi_{k}\right) \cdot \hat{\mathbf{n}}_{\partial \Omega} \mathrm{d} S \\
& \approx \sum_{n=1}^{I} \Psi_{k}\left(\mathbf{x}_{n}\right)\left[\left(U_{x_{1}}\left(\mathbf{x}_{n}\right)+k U\left(\mathbf{x}_{n}\right)\right)\left(x_{2, n}-x_{2, n-1}\right)\right. \\
& \left.\quad+\left(U_{x_{2}}\left(\mathbf{x}_{n}\right)+\mathrm{j} k U\left(\mathbf{x}_{n}\right)\right)\left(x_{1, n-1}-x_{1, n}\right)\right] \\
& =\sum_{n=1}^{I} \Psi_{k}\left(\mathbf{x}_{n}\right)\left\{\left(x_{2, n}-x_{2, n-1}\right) U_{x_{1}}\left(\mathbf{x}_{n}\right)+\left(x_{1, n-1}-x_{1, n}\right) U_{x_{2}}\left(\mathbf{x}_{n}\right)\right. \\
& \left.\quad+U\left(\mathbf{x}_{n}\right)\left[\left(x_{2, n}-x_{2, n-1}\right)+\mathrm{j}\left(x_{1, n-1}-x_{1, n}\right)\right]\right\} .
\end{aligned}
$$

The first term in (11) depends on $U\left(\mathbf{x}_{n}\right)$ and its spatial derivative which must be approximated from spatiotemporal samples of $u(\mathbf{x}, t)$. Note that $U\left(\mathbf{x}_{n}\right)$, can be obtained by sensor $n$ independently using trapezium rule. However, $\nabla U\left(\mathbf{x}_{n}\right)$ can only be estimated reliably using neighboring sensor measurements by a polynomial fitting approach. Specifically, we find the regression function $U\left(\mathbf{x}_{n}\right)=\alpha_{n} x_{1, n}+\beta_{n} x_{2, n}+\gamma_{n}$ by estimating $\left(\alpha_{n}, \beta_{n}, \gamma_{n}\right)$ for each boundary sensor $n=1, \ldots, I$ using measurements of the nearest neighbors to the point $\mathbf{x}_{n}$.

Let the $n$-th sensor located at $\mathbf{x}_{n}$, with measurement $U\left(\mathbf{x}_{n}\right)$ have the two closest sensors $\mathbf{x}_{n}^{\prime}$ and $\mathbf{x}_{n}^{\prime \prime}$ with corresponding measurements $U\left(\mathbf{x}_{n}^{\prime}\right)$ and $U\left(\mathbf{x}_{n}^{\prime \prime}\right)$. With these we can estimate the parameters $\left(\alpha_{n}, \beta_{n}, \gamma_{n}\right)$ by solving the linear system:

$$
\begin{aligned}
{\left[\begin{array}{c}
U\left(\mathbf{x}_{n}^{\prime \prime}\right) \\
U\left(\mathbf{x}_{n}\right) \\
U\left(\mathbf{x}_{n}^{\prime}\right)
\end{array}\right] } & =\left[\begin{array}{lll}
x_{1, n}^{\prime \prime} & x_{2, n}^{\prime \prime} & 1 \\
x_{1, n} & x_{2, n} & 1 \\
x_{1, n}^{\prime} & x_{2, n}^{\prime} & 1
\end{array}\right]\left[\begin{array}{c}
\alpha_{n} \\
\beta_{n} \\
\gamma_{n}
\end{array}\right], \\
\mathbf{u}_{n} & =\mathbf{X}_{n} \mathbf{d}_{n} .
\end{aligned}
$$

The system admits a unique solution, if $\mathbf{x}_{n}^{\prime \prime}, \mathbf{x}_{n}$ and $\mathbf{x}_{n}^{\prime}$ are not collinear. Therefore, the local spatial derivative $\nabla U\left(\mathbf{x}_{n}\right)$ can be retrieved directly from the solution to this system by noticing the polynomial $p(\mathbf{x})=\alpha x_{1}+\beta x_{2}+\gamma$ has $\nabla p(\mathbf{x})=\langle\alpha, \beta\rangle$. Hence $\nabla U\left(\mathbf{x}_{n}\right)=\left[U_{x_{1}}\left(\mathbf{x}_{n}\right), U_{x_{2}}\left(\mathbf{x}_{n}\right)\right]^{\top} \approx\left[\alpha_{n}, \beta_{n}\right]^{\top}$, where

$$
\begin{aligned}
& \alpha_{n}=\frac{\left(x_{2, n}-x_{2, n}^{\prime}\right) U\left(\mathbf{x}_{n}^{\prime \prime}\right)+\left(x_{2, n}^{\prime}-x_{2, n}^{\prime \prime}\right) U\left(\mathbf{x}_{n}\right)+\left(x_{2, n}^{\prime \prime}-x_{2, n}\right) U\left(\mathbf{x}_{n}^{\prime}\right)}{\operatorname{det}\left(\mathbf{X}_{n}\right)}, \\
& \beta_{n}=\frac{\left(x_{1, n}^{\prime}-x_{1, n}\right) U\left(\mathbf{x}_{n}^{\prime \prime}\right)+\left(x_{1, n}^{\prime \prime}-x_{1, n}^{\prime}\right) U\left(\mathbf{x}_{n}\right)+\left(x_{1, n}-x_{1, n}^{\prime \prime}\right) U\left(\mathbf{x}_{n}^{\prime}\right)}{\operatorname{det}\left(\mathbf{X}_{n}\right)} .
\end{aligned}
$$

Then substituting these back into (11) gives:

$$
\begin{aligned}
& \oint_{\partial \Omega}\left(\Psi_{k} \nabla U-U \nabla \Psi_{k}\right) \cdot \hat{\mathbf{n}}_{\partial \Omega} \mathrm{d} S \\
& \quad \approx \sum_{n=1}^{I} b_{n}^{\prime \prime}(k) U\left(\mathbf{x}_{n}^{\prime \prime}\right)+b_{n}(k) U\left(\mathbf{x}_{n}\right)+b_{n}^{\prime}(k) U\left(\mathbf{x}_{n}^{\prime}\right),
\end{aligned}
$$




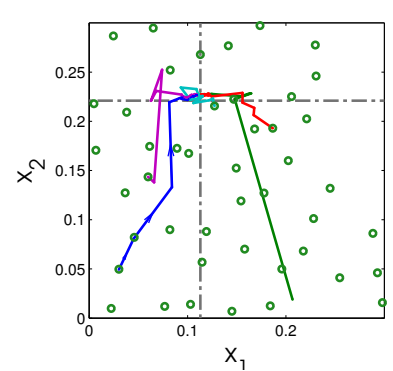

(a) Evolution of $\boldsymbol{\xi}_{1}$ est.

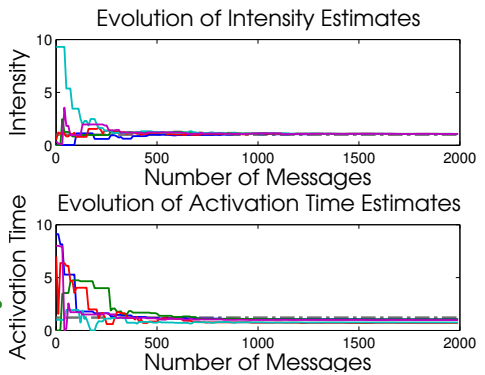

(b) Evolution of $c_{1}$ and $\tau_{1}$ ests.
Fig. 2: The distributed estimation algorithm for $M=1$ in the noiseless setting using 45 sensors. $c_{1}=1, \boldsymbol{\xi}_{1}=(0.113,0.221)$ and and $\tau_{1}=1.213 \mathrm{~s}$. Field is sampled for $T_{\text {end }}=10 \mathrm{~s}$ at $\frac{1}{\Delta t}=1 \mathrm{~Hz}$ with $K=1$ for sensing function.
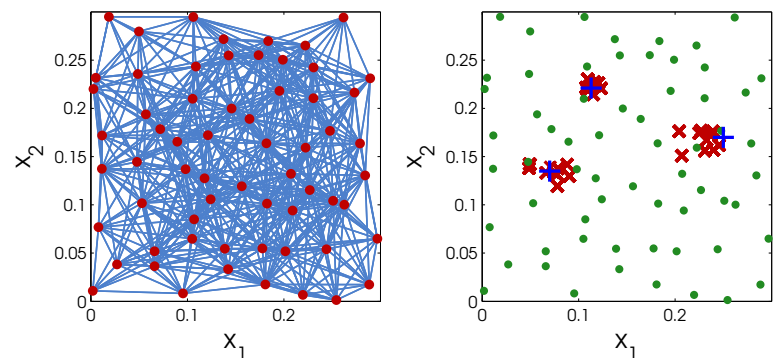

(a) A realization of the RGG (left) and $\boldsymbol{\xi}_{m}$ ests (right).

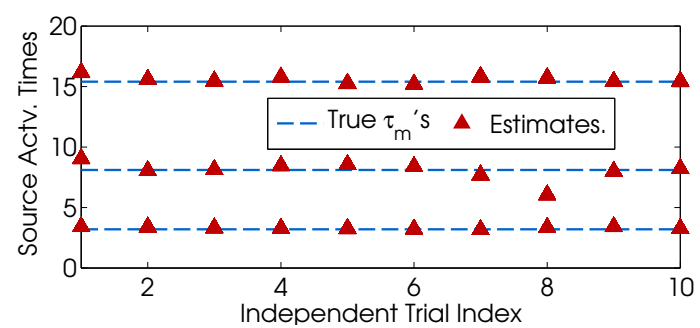

(b) Activation Time estimates.

Fig. 3: Distributed estimation of $M=3$ sources using 63 sensors. Sensor measurements have $\mathrm{SNR}=20 \mathrm{~dB} . c_{1}=c_{2}=c_{3}=1$; $\boldsymbol{\xi}_{1}=(0.113,0.221), \boldsymbol{\xi}_{2}=(0.250,0.170), \boldsymbol{\xi}_{3}=(0.070,0.135)$; and $\tau_{1}=3.2 s, \tau_{2}=8.1 s, \tau_{3}=15.4 \mathrm{~s}$. Field is sampled for $T_{\text {end }}=25 \mathrm{~s}$ at $\frac{1}{\Delta t}=2 \mathrm{~Hz}$ and $\mathrm{K}=10$ for sensing function. Scatter-plot shows the true source locations (blue ' + '), the estimated locations (red ' $X$ ') and one realization of the sensor distribution (green ‘ $\bullet$ ')

where

$$
\begin{aligned}
b_{n}^{\prime \prime}(k) & =\frac{\Psi_{k}\left(\mathbf{x}_{n}\right)}{\operatorname{det}\left(\mathbf{X}_{n}\right)}\left[\begin{array}{l}
x_{1, n-1}-x_{1, n} \\
x_{2, n}-x_{2, n-1}
\end{array}\right]^{\top}\left[\begin{array}{l}
x_{1, n}^{\prime}-x_{1, n} \\
x_{2, n}-x_{2, n}^{\prime}
\end{array}\right], \\
b_{n}(k) & =\frac{\Psi_{k}\left(\mathbf{x}_{n}\right)}{\operatorname{det}\left(\mathbf{X}_{n}\right)}\left[\begin{array}{l}
x_{1, n-1}-x_{1, n} \\
x_{2, n}-x_{2, n-1}
\end{array}\right]^{\top}\left[\begin{array}{l}
x_{1, n}^{\prime \prime}-x_{1, n}^{\prime} \\
x_{2, n}^{\prime}-x_{2, n}^{\prime \prime}
\end{array}\right] \\
& +k \Psi_{k}\left(\mathbf{x}_{n}\right)\left[\left(x_{2, n}-x_{2, n-1}\right)+\mathrm{j}\left(x_{1, n-1}-x_{1, n}\right)\right], \\
b_{n}^{\prime}(k) & =\frac{\Psi_{k}\left(\mathbf{x}_{n}\right)}{\operatorname{det}\left(\mathbf{X}_{n}\right)}\left[\begin{array}{l}
x_{1, n-1}-x_{1, n} \\
x_{2, n}-x_{2, n-1}
\end{array}\right]^{\top}\left[\begin{array}{l}
x_{1, n}-x_{1, n}^{\prime \prime} \\
x_{2, n}^{\prime \prime}-x_{2, n}
\end{array}\right] .
\end{aligned}
$$

The terms $b_{n}^{\prime \prime}(k), b_{n}(k)$ and $b_{n}^{\prime}(k)$ in (15) are dependent only on the topology of the network (specifically the locations of the sensors) and our choice of sensing function $\Psi_{k}(\mathbf{x})$. Indeed given the assumptions detailed in Section 2.1, these weights can be precomputed for every sensor in the network, such that

$$
\oint_{\partial \Omega}\left(\Psi_{k} \nabla U-U \nabla \Psi_{k}\right) \cdot \hat{\mathbf{n}}_{\partial \Omega} \mathrm{d} S \approx \sum_{n \in \mathcal{S}} B_{n}(k) U\left(\mathbf{x}_{n}\right),
$$

where $B_{n}(k)$ is non-zero if $n$ is a boundary sensor, i.e. $n \in \mathcal{S}_{\partial \Omega}$, or if it is one of the two nearest sensors to a boundary sensor, $n \in \mathcal{N}_{\partial \Omega}$. Otherwise $B_{n}(k)$ is zero. Finally, we can combine (10) and (16), to obtain the estimates for $\mathcal{R}(k)$ :

$$
\begin{aligned}
\mathcal{R}(k) & \approx \sum_{n \in \mathcal{S}} A_{n}(k) \dot{\Phi}_{n}\left(t_{L}\right)-\mu B_{n}(k) \Phi_{n}\left(t_{L}\right) \\
& =\frac{1}{N} \sum_{n \in \mathcal{S}} y_{n}(k)
\end{aligned}
$$

Upon deployment of the sensors, each sensor can precompute its unique weights $A_{n}(k)$ and $B_{n}(k)$ for $k=0, \ldots, K$. After which they can start to monitor the region of interest $\Omega$, by sensing the field locally. To initiate the estimation process, the sensor $n$ exchanges its modified measurements $\left\{y_{n}(k)\right\}_{k}$ with a randomly chosen neighbor. This begins the gossip round, as detailed in Section 3, it continues until convergence to $\{\mathcal{R}(k)\}$. All sensors in the network can now independently apply Prony's method to its current estimate of $\mathcal{R}(k)$ to recover all $M$ triples $\left\{c_{m}, \tau_{m}, \boldsymbol{\xi}_{m}\right\}$ as described in Section 4.1.

\section{SIMULATION RESULTS}

In Figure 2 we plot the trajectories of the source parameter estimates of five randomly chosen sensors, as the number of pairwise local message exchanges increases. Notice that the estimates converge to the desired values after several pairwise exchanges as expected. Furthermore, we investigate the robustness of the algorithm to noisy sensor measurements in the multiple source case. We perform 10 independent trials, with each trial using a new realization of a random geometric graph (RGG) and a new sensor noise process. As can be seen in Figures $3 \mathrm{a}$ and $3 \mathrm{~b}$, the source locations and activation times are recovered reliably even in the noisy setting, after $\sim 1000$ iterations. The intensity estimates are also retrieved, lying between 0.81-1.33 for each noisy independent trial.

\section{CONCLUSION}

We derived a fully distributed scheme for estimating the sources of a 2-D diffusion field from its arbitrary spatiotemporal samples. Specifically we solve the problem for jointly estimating all the unknown source parameters when the sources are localized and instantaneous. Then we extend this solution and propose a distributed algorithm based on average consensus schemes, when we have access only to discrete sensor network measurements. Finally we show through numerical simulations that the proposed algorithm is robust to noise, even in the multiple source setting.

\section{REFERENCES}

[1] A. Nehorai, B. Porat, and E. Paldi, "Detection and localization of vapor-emitting sources," IEEE Transactions on Signal Processing, vol. 43, no. 1, pp. 243-253, Jan 1995. 
[2] Y.M. Lu and M. Vetterli, "Spatial super-resolution of a diffusion field by temporal oversampling in sensor networks.," in Proc. of the 34th International Conference on Acoustics, Speech, and Signal Processing (ICASSP'09), Taipei, Taiwan, 2009, IEEE, pp. 2249-2252.

[3] I. Dokmanic, J. Ranieri, A. Chebira, and M. Vetterli, "Sensor networks for diffusion fields: Detection of sources in space and time," in Proc. 49th Annual Allerton Conference on Communication, Control, and Computing (Allerton'11), Sept 2011, pp. 1552-1558.

[4] J. Ranieri, A. Chebira, Y. M. Lu, and M. Vetterli, "Sampling and reconstructing diffusion fields with localized sources," in 36th IEEE International Conference on Acoustics, Speech and Signal Processing (ICASSP'11). IEEE, 2011, pp. 4016-4019.

[5] Y.M. Lu, P.L. Dragotti, and M. Vetterli, "Localization of diffusive sources using spatiotemporal measurements," in 49th Annual Allerton Conference on Communication, Control, and Computing (Allerton'11). IEEE, 2011, pp. 1072-1076.

[6] T. van Waterschoot and G. Leus, "Static field estimation using a wireless sensor network based on the finite element method," in 2011 4th IEEE International Workshop on Computational Advances in Multi-Sensor Adaptive Processing (CAMSAP'11), Dec 2011, pp. 369-372.

[7] J. Ranieri, I. Dokmanic, A. Chebira, and M. Vetterli, "Sampling and Reconstruction of Time-Varying Atmospheric Emissions," in Proc. of the 37th International Conference on Acoustics, Speech, and Signal Processing (ICASSP'12), Kyoto, Japan, 2012, IEEE.

[8] Y.M. Lu and M. Vetterli, "Distributed spatio-temporal sampling of diffusion fields from sparse instantaneous sources," in 3rd IEEE International Workshop on Computational Advances in Multi-Sensor Adaptive Processing (CAMSAP'09). IEEE, 2009, pp. 205-208.

[9] T. van Waterschoot and G. Leus, "Distributed estimation of static fields in wireless sensor networks using the finite element method," in Proc. 37th IEEE International Conference on Acoustics, Speech and Signal Processing (ICASSP'12), Kyoto, Japan, March 2012, IEEE, pp. 2853-2856.

[10] G. Reise, G. Matz, and K. Grochenig, "Distributed field reconstruction in wireless sensor networks based on hybrid shiftinvariant spaces," IEEE Transactions on Signal Processing,, vol. 60 , no. 10 , pp. 5426-5439, 2012.

[11] A. Schmidt and J.M.F. Moura, "A distributed sensor fusion algorithm for the inversion of sparse fields," in Proc. of the 43rd Asilomar Conference on Signals, Systems and Computers (Asolimar'09). IEEE, 2009, pp. 1332-1336.

[12] J. Murray-Bruce and P. L. Dragotti, "Reconstructing diffusion fields sampled with a network of arbitrarily distributed sensors," in Proc. European Signal Processing Conference (EUSIPCO`14), Lisbon, Portugal, Sept. 2014.

[13] J. Murray-Bruce and P. L. Dragotti, "Estimating localized sources of diffusion fields using spatiotemporal sensor measurements," IEEE Transactions on Signal Processing (To Appear), 2015.

[14] J.N. Tsitsiklis, Problems in decentralized decision making and computation, Ph.D. thesis, Massachusetts Institute of Technology, 1984.

[15] A. G. Dimakis, S. Kar, J. M. F. Moura, M. G. Rabbat, and A. Scaglione, "Gossip algorithms for distributed signal processing," Proceedings of the IEEE, vol. 98, no. 11, pp. 18471864, Nov 2010.

[16] S. Boyd, A. Ghosh, B. Prabhakar, and D. Shah, "Randomized gossip algorithms," IEEE Transactions on Information Theory, vol. 52, no. 6, pp. 2508-2530, 2006.

[17] T.C. Aysal, M.E. Yildiz, A.D. Sarwate, and A. Scaglione, "Broadcast gossip algorithms for consensus," IEEE Transactions on Signal Processing, vol. 57, no. 7, pp. 2748-2761, 2009.

[18] F. Bénézit, A.G. Dimakis, P. Thiran, and M. Vetterli, "Gossip along the way: Order-optimal consensus through randomized path averaging," in Proceedings of the Allerton Conference on Communication, Control, and Computing, 2007.

[19] J. Murray-Bruce and P. L. Dragotti, "Spatio-Temporal sampling and reconstruction of diffusion fields induced by point sources," in Proc. 39th IEEE International Conference on Acoustics, Speech and Signal Processing (ICASSP'14), Florence, Italy, May 2014, pp. 31-35.

[20] P.-L. Dragotti, M. Vetterli, and T. Blu, "Sampling moments and reconstructing signals of finite rate of innovation: Shannon meets strang-fix," IEEE Transactions on Signal Processing, vol. 55, no. 5, pp. 1741-1757, 2007.

[21] P. Stoica and R.L. Moses, Introduction to spectral analysis, Prentice Hall, Englewood Cliffs, NJ, 1997.

[22] K. Georg, "Approximation of integrals for boundary element methods," SIAM Journal on Scientific and Statistical Computing, vol. 12, no. 2, pp. 443-453, 1991. 